\title{
Prevalence of NRAS, PTEN and AKT1 gene mutations in the central nervous system metastases of non-small cell lung cancer
}

\author{
Marcin Nicoś ${ }^{1} \cdot$ Pawel Krawczyk $^{1}$ - Bożena Jarosz $^{2} \cdot$ Marek Sawicki $^{3}$. \\ Tomasz Trojanowski ${ }^{2} \cdot$ Janusz Milanowski $^{1}$
}

Received: 21 November 2016 / Accepted: 23 December 2016 / Published online: 17 January 2017

(C) The Author(s) 2017. This article is published with open access at Springerlink.com

\begin{abstract}
Somatic mutations in NRAS, PTEN and AKTI genes are rarely $(\sim 1 \%)$ reported in primary NSCLC, but their role in carcinogenesis have been proven. Therefore, we assessed the frequency of them in 145 FFPE tissue samples from CNS metastases of NSCLC using the real-time PCR technique. We identified four (two NRAS and single $A K T 1$ and PTEN) mutations in CNS metastases of NSCLC. All mutations were observed in current male smokers (4\% out of the male group; $4 / 100$ and $4.25 \%$ out of smokers; 4/94). Three mutations have been detected in patients with SqCC (10.3\% out of SqCC patients; 3/29), and only one mutation in the NRAS gene-in a patient with adenocarcinoma (1.25\% out of AC patients; $1 / 80)$. The examined genes were mutually exclusive in terms of molecular background in KRAS; EGFR; DDR2; PIK3CA; HER2 and $M E K 1$ genes that were evaluated in our previous studies. The OS of the patients who harbored NRAS, AKTI and PTEN mutations was 10.1, 12.1, 7.3 and 4 months, respectively (vs 13.5 months of the studied group). Our results suggest that the presence of NRAS, PTEN and AKTl gene mutations may have an influence on the occurrence of CNS metastases in patients with $\mathrm{SqCC}$.
\end{abstract}

Marcin Nicoś

marcin_nicos@interia.pl

1 Department of Pneumonology, Oncology and Allergology, Medical University of Lublin, Jaczewskiego 8, 20-954 Lublin, Poland

2 Pathological Laboratory, Department of Neurosurgery and Pediatric Neurosurgery, Medical University of Lublin, 20-954 Lublin, Poland

3 Department of Thoracic Surgery, Medical University of Lublin, 20-954 Lublin, Poland
Keywords $A K T 1 \cdot P T E N \cdot N R A S \cdot$ NSCLC $\cdot$ Central nervous system metastases

$\begin{array}{ll}\text { Abbreviations } \\ \text { AC } & \text { Adenocarcinoma } \\ \text { CE-IVD } & \text { Certified for in vitro diagnosis } \\ \text { CNS } & \text { Central nervous system } \\ \text { FFPE } & \text { Formalin-fixed paraffin-embedded } \\ \text { NSCLC } & \text { Non-small cell lung cancer } \\ \text { mt } & \text { Mutant types } \\ \text { OS } & \text { Overall survival } \\ \text { SqCC } & \text { Squamous cell carcinoma } \\ \text { TKIs } & \text { Tyrosine kinase inhibitors } \\ \text { wt } & \text { Wild type }\end{array}$

\section{Bacground}

Among metastatic sites of non-small cell lung cancer (NSCLC) central nervous system (CNS) lesions occur in $20-40 \%$ of lung adenocarcinoma (AC) patients and they are associated with neurological symptoms and extremely poor survival prognosis. In squamous cell carcinoma (SqCC), CNS metastases are observed less frequently (10-15\%) [1-4]. In patients with CNS metastases, the administration of standard chemotherapies or targeted agents is limited because of uncertain penetration of anticancer drugs through the blood-brain barrier and poor prognosis [5-8].

Today, we have found that mutational deregulations of pro-survival (PI3K-mTOR-AKT) and proliferative (RasRaf-Mek-Erk) cascades play a crucial role in uncontrolled signal transduction in cancer cells [9-11]. The majority of mutations involved in NSCLC carcinogenesis were reported in five oncogenes: KRAS, EGFR, ALK, HER2 and $B R A F[10,12]$. However, the presence of mutations in 
NRAS, MEK, AKT1, PTEN, RET, ROS1 genes had identified impact on acquiring resistance to both EGFR or ALK TKIs and radiotherapy $[9,13]$. Some papers reported that the MEK (selumetinib and trametinib), IGF-1R (linsitinib) or allosteric PI3K (LY294002) inhibitors may become an attractive therapeutic choice for NSCLC patients with some rare mutations [4, 9-11, 14-16].

To date, the majority of published data evaluated gene mutations in primary tumors of NSCLC. However, there is still limited data assessing genetic disorders in metastatic lesions. In our previous study, we focused on commonly mutated genes playing role in carcinogenesis. Therefore, the following study, we evaluated the prevalence of NRAS, $P T E N$ and $A K T 1$ gene mutations in Caucasian patients with CNS metastases of NSCLC.

\section{Methods}

\section{Patients}

The studied group included 145 Polish NSCLC patients with CNS metastases of advanced NSCLC. In 30 patients, the corresponding primary tumors were simultaneously available. The patients underwent routine neurosurgical procedures with a palliative manner. In the moment of CNS metastases diagnosis, all patients were chemo-, radio- or targeted therapy naïve. They did not receive any other treatment which could affect mutation inducement. The median overall survival (OS) was 13.5 months (range 0.1-78.2 months-information available from 119 patients). Detailed characteristics of the studied group have been presented in Table 1. DNA was isolated from formalin-fixed paraffin-embedded (FFPE) tissue samples using the QIAamp DNA FFPE Tissue Kit (Qiagen, USA) according to manufacturer protocol. The positive control of the analysis was the reaction with control DNA supplied with the assay by the manufacturer. DNA isolated from peripheral blood leukocytes of healthy individuals $(n=10)$ provide the negative control of analysis. The study was approved by the Ethics Committee of the Medical University of Lublin, Poland (No. KE-0254/86/2013). All patients expressed their consent to participate in the study and they expressed their consent to publish their individual data (if it is needed).

\section{NRAS mutation analysis}

NRAS gene status was evaluated using the NRAS Mutation Analysis Kit (EntroGen, USA) certified for in vitro diagnosis (CE-IVD). This kit determines 12 substitutions (G12D, G12S, G12C, G13R, G13V, Q61K, Q61L, Q61R, Q61H, A126T, K117R, A59X) located in exons 2, 3 and 4 of the
Table 1 Detailed studied group characteristics

\begin{tabular}{ll}
\hline Gender & \\
Male, $n(\%)$ & $100(69)$ \\
Female, $n(\%)$ & $45(31)$ \\
Age & \\
Median age \pm SD (years) & $60 \pm 8.8$ \\
$\quad \geq 60$ years, $n(\%)$ & $72(49.7)$ \\
$\quad<60$ years, $n(\%)$ & $73(50.3)$ \\
Histopathology & \\
Adenocarcinoma, $n(\%)$ & $80(55.2)$ \\
Squamous cell carcinoma, $n(\%)$ & $29(20)$ \\
Large-cell carcinoma, $n(\%)$ & $22(15.1)$ \\
NSCLC-NOS, $n(\%)$ & $14(9.7)$ \\
Smoking status & \\
Current smokers, $n(\%)$ & $73(50.4)$ \\
Former smokers, $n(\%)$ & $21(14.5)$ \\
Non-smokers, $n(\%)$ & $36(24.8)$ \\
Lack of data, $n(\%)$ & $15(10.3)$ \\
Performance status $(\mathrm{ECOG} / \mathrm{WHO})$ & \\
$0, n(\%)$ & $22(15.2)$ \\
$1, n(\%)$ & $76(52.4)$ \\
$2, n(\%)$ & $31(21.4)$ \\
$3, n(\%)$ & $16(11)$ \\
\hline
\end{tabular}

$N R A S$ gene. The amplification of the examined region was performed in 96-well plates in a real-time PCR device (Cobas, Roche, USA) in the following steps: pre-denaturation in $95^{\circ} \mathrm{C}$ for $10 \mathrm{~min}$. and 40 cycles in conditions: $95^{\circ} \mathrm{C}$ for $15 \mathrm{~s}$. and $60^{\circ} \mathrm{C}$ for $40 \mathrm{~s}$.

\section{PTEN and AKT1 mutation analysis}

PTEN and AKT1 gene mutations were evaluated using the TaqMan Mutation Detection Assay (Applied Biosystem, USA) certified for research use. The amplification of examined genes was performed in 96-well plates in a realtime PCR device (Cobas, Roche, USA) in the steps recommended by Applied Biosystems: $95^{\circ} \mathrm{C}$ for $10 \mathrm{~min}$ and $92^{\circ} \mathrm{C}$ for $15 \mathrm{~s}, 58^{\circ} \mathrm{C}$ for $1 \mathrm{~min}$. for five cycles then $92^{\circ} \mathrm{C}$ for $15 \mathrm{~s}, 60^{\circ} \mathrm{C}$ for $60 \mathrm{~s}$ for 40 cycles.

\section{Results analysis}

Fluorescence was observed only during amplification of mutant types (mt) in analyzed samples and in the endogenous control. According to observations made of the positive and negative control amplification plots, samples were assessed as $\mathrm{mt}$ if we observed amplification between 25 and 30 cycles. The samples without or with late amplification $\left(C_{t}>35\right.$ cycle) were assessed as wild type (wt). Based on amplification curves in $\mathrm{mt}$ samples and the corresponding 
endogenous wt control, we estimated the frequency of $\mathrm{mt}$ DNA according to the following equation:

$\%$ mutated DNA $=2^{-\Delta C t} \times 100 \%$

$\Delta C t$ (analyzed sample) $=$ the average $C t$ value from the mutant reaction-the average $C t$ value from the wild-type reaction.

\section{Results}

We identified four (two NRAS and single AKT1 and PTEN) mutations in CNS metastases of NSCLC. The content of $\mathrm{mt}$ allele in all mutated samples was $>5 \%$. All mutations were observed in current male smokers ( $4 \%$ out of the male group; $4 / 100$ and $4.25 \%$ out of smokers; $4 / 94)$. Three mutations have been detected in patients with SqCC $(10.3 \%$ out of SqCC patients; 3/29), and only one mutation in the $N R A S$ gene-in a patient with adenocarcinoma $(1.25 \%$ out of AC patients; 1/80). Slides presenting histopathology differentiation for patients with detected mutation were presented at Fig. 1. The examined genes were mutually exclusive in terms of molecular background in KRAS; EGFR;
DDR2; PIK3CA; HER2 and MEK1 genes that were evaluated in our previous studies [17-22]. A simultaneous evaluation of 30 patients in whom both CNS metastases and the corresponding primary tumors were available, showed the presence of wt in NRAS, AKTI and PTEN genes in both lesions. Unfortunately, the corresponding primary tumors were unavailable from patients who harbored NRAS, AKTI and PTEN mutations in CNS metastases. Due to low quality of DNA and sub-clonality of CNS metastases we did not perform deep sequencing approaches to confirm our results.

Using an EntoGen kit we identified Q61L and A126T substitutions in the NRAS gene in two patients $(1.4 \%$ out of all patients; 2/145). A Q61L substitution was observed in a 47-year-old patient (35 pack-years) with AC histology (1.25\% out of AC patients; 1/80); while an A126T substitution was found in a 71-year-old patient (20 pack-years) with squamous cell carcinoma histology (3.5\% out of SqCC patients; 1/29). The overall survival (OS) of NRAS mutated patients was 10.1 and 12.1 months, respectively.

Using TaqMan hydrolysis probes we detected an E17K substitution in the $A K T 1$ gene ( $0.7 \%$ of studied group) and an R233X substitution in the PTEN gene $(0.7 \%$ of the
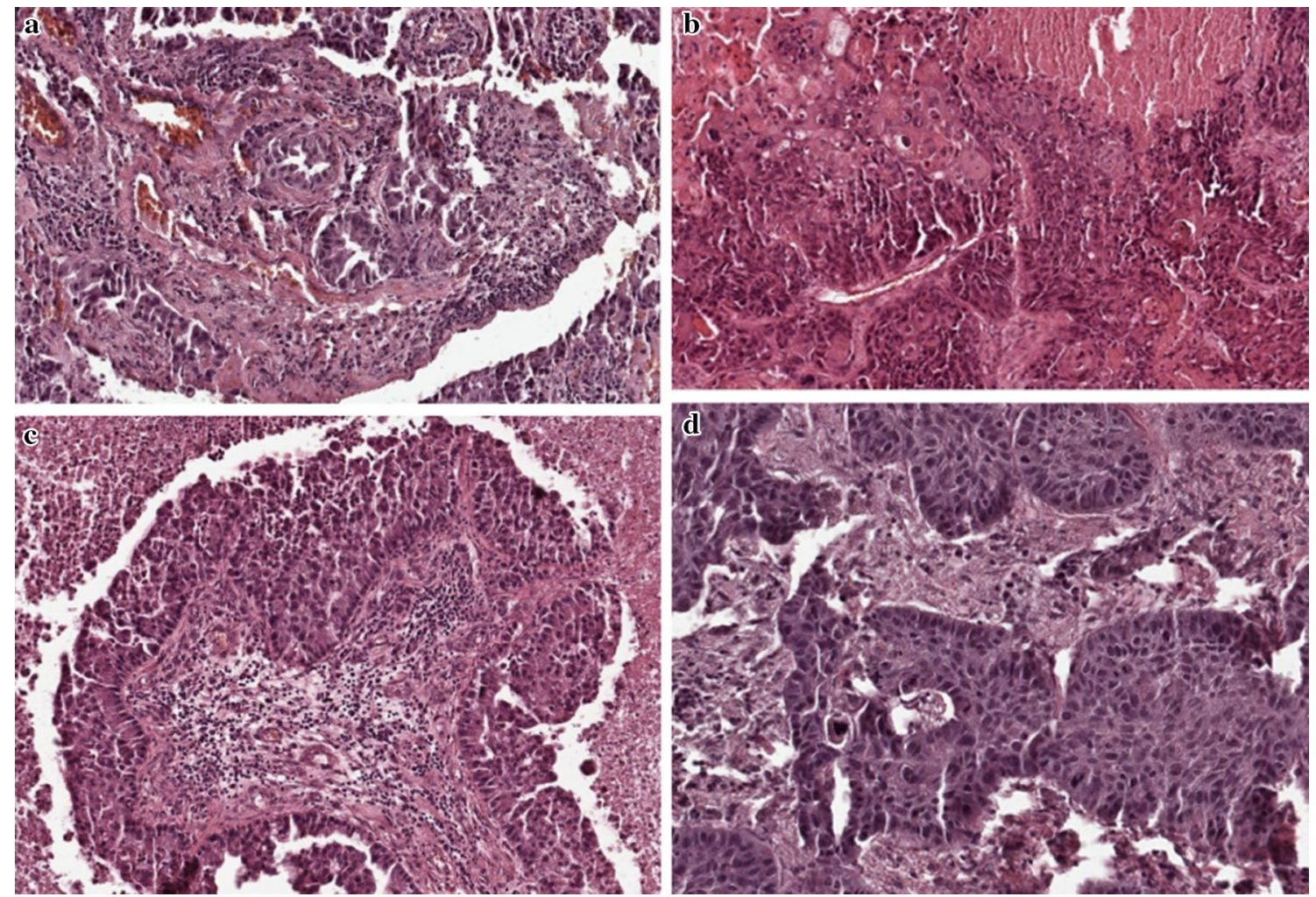

Fig. 1 Slides presenting histopathology differentiation of patients with detected mutations. a Shows adenocarcinoma type of NSCLC in patients with Q61L substitution in NRAS gene. b Shows squamous cell carcinoma type of NSCLC in patients with A126T substitution

in NRAS gene. c Shows squamous cell carcinoma type of NSCLC in patients with E17K substitution in $A K T 1$ gene. d Shows squamous cell carcinoma type of NSCLC in patients with R233X substitution in PTEN gene 
studied group). The AKT1 gene mutation was observed in a 73-year-old patient (20 pack-years) with SqCC histology (3.5\% out of SqCC patients; 1/29). The PTEN gene mutation was found in a 62-year-old patient (50 pack-years) with SqCC histology (3.5\% out of SqCC patients; $1 / 29)$. The OS of $A K T 1$ and PTEN mutated patients was 7.3 and 4 months, respectively.

The summary of clinical and demographical data of positive patients has been presented in Table 2 .

\section{Discussion}

Brain metastases are one of the most common metastatic lesions of NSCLC which are associated with a high mortality of patients [1-4]. Moreover, a blood-brain barrier ensures restrict transit of agents into the brain parenchyma, which are considered as pharmacological sanctuary lesions that show limited sensitivity to anti-cancer therapy $[1,2]$. However, there are some studies which indicated the effectiveness of anti-ALK targeted therapies (alectinib and ceritinib) also in CNS metastatic sites of NSCLC [6, 23]. Till today, we have only limited data concerning the evaluation of the most frequent mutations in $E G F R, K R A S$, $B R A F$ genes in CNS metastases of lung cancer (especially AC type). Despite NRAS, PTEN and AKT1 mutations have proven involvement in carcinogenesis [24-28], their frequency was described in a few reports only in primary NSCLC tumors [3, 4, 13, 14, 16]. Therefore, we performed the current and unique characteristic of the incidence of NRAS, AKTI and PTEN gene mutations in CNS metastases of NSCLC.

\section{NRAS gene mutations in NSCLC patients}

NRAS as a member of the RAS family plays a role in the MAPK signaling pathway and its deregulation can lead to tumorgenesis [14, 29, 30]. Activating mutations in exons 2 (codons 12 and 13), 3 (codons 59 and 61) and 4 (codons 117,126 and 146) of the NRAS gene have been frequently described in melanoma (13-25\%), myeloid leukemia (10\%), colorectal cancer (2-5\%), hepatocellular carcinoma (1.4\%) and thyroid carcinoma (6\%) [31]. Among all well-known NRAS activating mutations, the substitutions in codon 61 are more frequent $(90 \%)$ than substitutions in other codons $[10,14,30]$. The most common transversions are described as $\mathrm{G}>\mathrm{C}$ and $\mathrm{T}>\mathrm{A}$. It was previously reported that air fossil fuel pollution (including di-methylobenza-anthracene) are involved in the induction of $\mathrm{A}>\mathrm{T}$ and $\mathrm{T}>\mathrm{A}$ changes. Moreover, the combination of smoking and environmental carcinogens can be associated with the etiology of NRAS mutated lung cancer [14, 32].

In our analysis, we identified two NRAS mutations in CNS metastases of NSCLC including one Q61L substitution, which is reported in the literature as the most frequent type $[10,14,30,31]$. The A126T substitution was the second NRAS mutation which is described as extremely rare $[10,14,30]$. To date, only Preusser et al. identified one $(1.3 \% ; 1 / 76)$ NRAS mutation in brain metastases of lung AC [4]. Most of comprehensive analyses that were carried out in the primary NSCLC, reported an extremely rare frequency $(<1 \%)$ of NRAS mutation and described them as related to AC type and current smoking status [12, 14, 28]. Moreover, Ohashi et al. observed overlapping of one NRAS mutation with KRAS G12A substitution and another one with $M E T$ gene amplification [12, 14, 24].

\section{AKT1 gene mutation in NSCLC patients}

AKT1 promotes the PI3K signaling pathway and is involved in cells proliferation and motility [9, 29]. A main E17K substitution in the $A K T 1$ gene was first identified by Carpten et al. in 2007 in breast cancers (8\%), and to date, it was also reported in other solid tumors: colorectal $(6 \%)$ and bladder cancers $(5 \%)$ [10, 25].

The following analysis is the first report worldwide that describes one E17K substitution in the $A K T 1$ gene in CNS metastases of NSCLC. The corresponding primary tumor from this patient showed a native form of the $A K T 1$ gene. It indicates that the molecular status of the $A K T 1$ gene could be changeable during disease progression and disturbances in the PI3K-mTOR-AKT pathway can be involved in the process of metastasis $[3,4,9]$. The frequency of $A K T 1$ mutation in primary NSCLC tumors is also reported as low $(<1 \%)$. These mutations occur more frequently in smokers and in SqCC [10, 12, 16, 24, 26, 27, 33-35]. However, Malanga et al. described the higher frequency of $A K T 1$ mutation $(1.9 \% ; 2 / 105)$ in NSCLC patients, especially in smokers and in SqCC type (5.5\%; 2/36). They also suggested that hyperactivation of AKT1 cascade (due to E17K
Table 2 The summary of clinical and demographic data of NSCLC patients with NRAS, AKT1 and PTEN gene mutations

\begin{tabular}{lllllll}
\hline Gene & Substitution & Histology & Age & Gender & Smoking (pack-years) & OS (months) \\
\hline \multirow{2}{*}{ NRAS } & Q61L & AC & 47 & M & Former (35) & 10.1 \\
& A126T & SqCC & 71 & M & Former (20) & 12.1 \\
AKT1 & E17K & SqCC & 73 & M & Former (20) & 7.3 \\
PTEN & R233X & SqCC & 62 & M & Current (50) & 4 \\
\hline
\end{tabular}


mutation) may be involved in the development of SqCC tumors [34].

\section{PTEN gene mutation in NSCLC patients}

Phosphatase and tensin homolog (PTEN) is described as a tumor suppressor gene whose product regulates a PI3KAKT-mTOR signaling cascade. The PTEN is involved in the stimulation of apoptosis, inhibition of cells' migration and regulation in both $\mathrm{p} 53$ protein levels and activity $[11,13,28]$. The most common PTEN abnormality was described as a loss of heterozygosity and promoter methylation of the PTEN gene ( $50 \%$ of NSCLC patients), however, some data also reported the presence of inactivating PTEN mutations [28, 36, 37].

In our analysis, we identified one inactivating PTEN gene mutation in CNS metastases of NSCLC and it is lower than PTEN mutation incidence reported in primary NSCLC $(\sim 4 \%)$. The previous literature data concerned the frequency of all inactivating PTEN mutations that were identified in NSCLC patients $[28,36]$. However, in the following study we focused on only one mutation (R233X substitution) for which there are some indications of its clinical significance [11, 15, 28, 36, 37]. Jin et al. and Lee et al. showed that in the primary NSCLC, PTEN mutations are related to smoking and SqCC types [28, 36], however, significant relations to other clinicopathologic factors, such as age, gender and degree of cell differentiation have not been reported $[28,36,37]$. Some studies also indicated overlapping of PTEN mutations with EGFR; ERBB2; KRAS and TP53 mutations $[15,28,37]$. In our study, all examined genes were mutually exclusive from $K R A S ; E G F R ; D D R 2$; PIK3CA; NRAS; HER2 and MEK1 genes.

\section{Conclusions}

In summary, particular NRAS, AKTI and PTEN gene mutations occur with similar rare $(\sim 1 \%)$ frequency in CNS metastases of NSCLC as in primary lung cancer tumors. Identification of the mutations is more likely in SqCC patients (especially in male smokers). Our results suggest that it is most likely to indicate the occurrence of NRAS, $A K T 1$ and PTEN mutations in metastatic sites of squamous cell lung carcinoma. An evaluation of the effectiveness of molecularly targeted agents in patients who harbor the mutations might be considered as a beneficial therapeutic choice in NSCLC patients with CNS metastases.

\section{Compliance with ethical standards}

Conflict of interest The authors declare that they have no competing interests.
Informed consent Informed consent was obtained from all individual participants included in the study. The research involved human participants. The study was approved by the Ethics Committee of the Medical University of Lublin, Poland (No. KE-0254/86/2013).

Open Access This article is distributed under the terms of the Creative Commons Attribution 4.0 International License (http:// creativecommons.org/licenses/by/4.0/), which permits unrestricted use, distribution, and reproduction in any medium, provided you give appropriate credit to the original author(s) and the source, provide a link to the Creative Commons license, and indicate if changes were made.

\section{References}

1. Renfrow JJ, Lesser GJ (2013) Molecular subtyping of brain metastases and implications for therapy. Curr Treat Options Oncol 14:514-527

2. Takei H, Rouah E, Ishida Y (2015) Brain metastasis: clinical characteristics, pathological findings and molecular subtyping for therapeutic implications. Brain Tumor Pathol. doi:10.1007/ s10014-015-0235-3

3. Villalva C, Duranton-Tanneur V, Guilloteau K et al (2013) EGFR, KRAS, BRAF, and HER-2 molecular status in brain metastases from 77 NSCLC patients. Cancer Med 2:296-304.

4. Preusser M, Berghoff AS, Koller R et al (2015) Spectrum of gene mutations detected by next generation exome sequencing in brain metastases of lung adenocarcinoma. Eur $\mathbf{J}$ Cancer 51:1803-1811

5. Welsh JW, Komaki R, Amini A et al (2013) Phase II trial of erlotinib plus concurrent whole-brain radiation therapy for patients with brain metastases from non-small-cell lung cancer. J Clin Oncol 31:895-902

6. Gadgeel SM, Gandhi L, Riely GJ et al (2014) Safety and activity of alectinib against systemic disease and brain metastases in patients with crizotinib-resistant ALK-rearranged non-small-cell lung cancer (AF-002JG): results from the dose-finding portion of a phase 1/2 study. Lancet Oncol 15:1119-1128

7. Kaneda H, Okamoto I, Nakagawa K (2013) Rapid response of brain metastasis to crizotinib in a patient with ALK rearrangement positive non-small-cell lung cancer. J Thorac Oncol 8:32-33

8. Robinson SD, O'Shaughnessy JA, Cowey CL et al (2014) BRAF V600E-mutated lung adenocarcinoma with metastases to the brain responding to treatment with vemurafenib. Lung Cancer $85: 326-330$

9. Vijayalakshmi R, Krishnamurthy A (2011) Targetable "driver" mutations in non small cell lung cancer. Indian. J Surg Oncol 2:178-188

10. Greulich H (2010) The genomics of lung adenocarcinoma: opportunities for targeted therapies. Genes Cancer 12:1200-1210

11. Nurwidya F, Takahashi F, Murakami A et al. (2014) Acquired resistance of non-small cell lung cancer to epidermal growth factor receptor tyrosine kinase inhibitors. Resp Invest 52: 82-91.

12. Kris MG, Johnson BE, Berry LD et al (2014) Using multiplexed assays of oncogenic drivers in lung cancers to select targeted drugs. JAMA 311:1998-2006

13. Huang L, Fu L (2015) Mechanisms of resistance to EGFR tyrosine kinase inhibitors. Acta Pharm Sin B 5:390-401.

14. Ohashi K, Sequist LV, Arcila ME et al (2013) Characteristics of lung cancers harboring NRAS mutations. Clin Cancer Res 19:2584-2591 
15. Su J, Zhang XC, An SJ et al (2014) Detecting the spectrum of multigene mutations in non-small cell lung cancer by Snapshot assay. Chin J Cancer 33:346-350

16. Do H, Solomon B, Mitchell PL et al (2008) A. Detection of the transforming AKT1 mutation E17K in non-small cell lung cancer by high resolution melting. BMC Res Notes. doi:10.1186/1756-0500-1-14

17. Kamila WK, Michał S, Paweł K et al (2013) EGFR activating mutations detected by different PCR techniques in Caucasian NSCLC patients with CNS metastases: short report. Clin Exp Metastasis 30:1063-1071

18. Nicoś M, Krawczyk P, Jarosz B et al (2016) Analysis of KRAS and BRAF genes mutation in the central nervous system metastases of non-small cell lung cancer. Clin Exp Med 16:169-176

19. Nicoś M, Powrózek T, Krawczyk P et al (2014) Sensitive methods for detection of the S768R substitution in exon 18 of the DDR2 gene in patients with central nervous system metastases of non-small cell lung cancer. Med Oncol. doi:10.1007/ s12032-014-0176-4

20. Nicoś M, Krawczyk P, Jarosz B et al (2016) Sensitive methods for screening of the MEK1 gene mutations in patients with central nervous system metastases of non-small cell lung cancer. Clin Transl Oncol. doi:10.1007/s12094-016-1483-3

21. Krawczyk P, Nicoś M, Powrózek T et al (2013) Sensitive methods for the detection of an insertion in exon 20 of the HER 2 gene in the metastasis of non-small cell lung cancer to the central nervous system. Oncol Lett 6:1063-1067.

22. Nicoś M, Krawczyk P, Powrózek T et al (2016) PIK3CA mutations detected in patients with central nervous system metastases of non-small cell lung cancer. Anticancer Res 36:2243-2249

23. Shaw AT, Kim DW, Mehra R et al (2014) Ceritinib in ALK-rearranged non-small-cell lung cancer. N Engl J Med 370:1189-1197

24. Cancer Genome Atlas Research Network (2014) Comprehensive molecular profiling of lung adenocarcinoma. Nature 511:543-550

25. Carpten JD, Faber AL, Horn C et al (2007) A transforming mutation in the pleckstrin homology domain of AKT1 in cancer. Nature 448:439-444
26. Kim MS, Jeong EG, Yoo NJ et al (2008) Mutational analysis of oncogenic AKT E17K mutation in common solid cancers and acute leukaemias. Br J Cancer 98:1533-1535

27. Bleeker FE, Felicioni L, Buttitta F et al (2008) AKT1(E17K) in human solid tumours. Oncogene 27:5648-5650

28. Jin G, Kim MJ, Jeon HS et al (2010) PTEN mutations and relationship to EGFR, ERBB2, KRAS, and TP53 mutations in nonsmall cell lung cancers. Cancer 69:279-283

29. Shimizu K, Goldfarb M, Suard Y et al (1983) Three human transforming genes are related to the viral ras oncogenes. Proc Natl Acad Sci USA 80:2112-2116

30. Ding L, Getz G, Wheeler DA et al (2008) Somatic mutations affect key pathways in lung adenocarcinoma. Nature 455:1069-7105

31. Vujic I, Posch C, Sanlorenzo M et al (2014) Mutant NRASQ61 shares signaling similarities across various cancer types-potential implications for future therapies. Oncotarget 17:7936-7944

32. Osaka M, Matsuo S, Koh T et al (1995) N-ras mutation in 7,12-dimethylbenz[a]anthracene (DMBA)-induced erythroleukemia in Long-Evans rats. Cancer Lett 91:25-31

33. Pao W, Girard N (2011) New driver mutations in non-small-cell lung cancer. Lancet Oncol 12:175-180

34. Malanga D, Scrima M, De Marco C, Fabiani F, De Rosa N et al (2008) Activating E17K mutation in the gene encoding the protein kinase AKT1 in a subset of squamous cell carcinoma of the lung. Cell Cycle 7:665-669

35. Cancer Genome Atlas Research Network (2012) Comprehensive genomic characterization of squamous cell lung cancers. Nature 489:519-525

36. Lee SY, Kim MJ, Jin G et al (2010) Somatic mutations in epidermal growth factor receptor signaling pathway genes in non-small cell lung cancers. J Thorac Oncol 11:1734-1740

37. Malkoski SP, Cleaver TG, Thompson JJ et al (2014) Role of PTEN in basal cell derived lung carcinogenesis. Mol Carcinog $53: 841-846$ 